\title{
Normokalemic Periodic Paralysis
}

National Cancer Institute

\section{Source}

National Cancer Institute. Normokalemic Periodic Paralysis. NCI Thesaurus. Code C122791.

An autosomal dominant inherited non-dystrophic myotonia caused by mutations of the SCN4A gene, resulting in sodium muscle channelopathy. Currently, it is considered a variant of hyperkalemic periodic paralysis. Patients with normokalemic periodic paralysis do not have any change in their potassium levels during weakness, but become weak when they ingest potassium. 